\title{
Giant Cervical Immature Teratoma: A Case Report of a Fetal Autopsy
}

\author{
Mozhgan Akbarzadeh-Jahromi ${ }^{1,}$; Fatemeh Sari Aslani ${ }^{1}$ \\ ${ }^{1}$ Department of Pathology, Shiraz University of Medical Sciences, Shiraz, IR Iran \\ *Corresponding Author: Mozhgan Akbarzadeh-Jahromi, Department of Pathology, Shiraz University of Medical Sciences, Shiraz, IR Iran. Tel: +98-9171207802, Fax: +98-7112301784, \\ E-mail: akbarzadeh@sums.ac.ir
}

Received: January 1, 2014; Revised: April 30, 2014; Accepted: June 1, 2014

\begin{abstract}
Introduction: Cervical teratoma is a rare neoplasm originating from the pluripotent cells and ectopic embryogenic non-germ cells and is manifested as a large and deformed mass in the neck.

Case presentation: We reported a fetal autopsy case with the diagnosis of cervical immature teratoma. Prenatal sonography showed a huge neck mass with differential diagnosis of thyromegaly or thymoma.

Conclusions: On autopsy, a well circumscribed encapsulated mass measuring $10 \times 7 \times 5$ centimeter was identified. Histopathological examination showed a neoplastic tissue composed of haphazardly arranged various mature and immature tissues derived from the three germ cell layers. The thyroid gland was not identified in the gross and serial sections of microscopic examination. Therefore teratoma must be considered in the differential diagnosis of neck masses in fetus and may involve the thyroid gland or arise from it.
\end{abstract}

Keywords:Immature Teratoma; Cervical; Fetus; Thyroid

\section{Introduction}

Cervical teratoma is a rare neoplasm originating from the pluripotent cells and ectopic embryogenic non germ cells and is manifested as a large and deformed mass in the neck (1). These tumors constitute about $35 \%$ of all teratomas, and its incidence varies from 1 in 20,000 to 1 in 40,000 , with a female-to-male ratio of 3:1(2).

\section{Case Presentation}

A female dead fetus with gestational age of 35 weeks after a normal vaginal delivery by a 27 year old mother gravid 1 was sent to our laboratory for autopsy. On sonography, polyhydramnios and a dead fetus with a huge mass in the anterior aspect of the neck were reported, with differential diagnosis of thyromegaly or thymoma. The external surface of the fetus was cyanotic with a large mass in the anterior aspect of the neck. The fetus was opened by Y incision, showing an encapsulated circumscribed mass with smooth external surface in the neck measuring $10 \times 7 \times 5$ $\mathrm{cm}$, which compressed the trachea and esophagus (Figure 1). On sectioning, a variegated surface with solid cystic area and foci of hemorrhage were visible. Grossly the thyroid gland was not identified. Other organs were without anomaly. Microscopic examination showed a neoplastic tissue composed of haphazardly arranged various mature and immature tissues containing islands of cartilage and bone, small islands of squamous epithelium without cytological malignancy, glands lined with tall columnar epithelium, smooth muscle fibers, adipose tissue and many blood vessels. Immature tissue was composed of sheets of small cells with hyperchromatic nuclei, in some areas with a neurofibrillary background forming rosettes (Figure $2 \mathrm{~A}$ and B). Thyroid tissue was not identified in the serial sections of neck mass and soft tissues of anterior aspect of the neck. Other organs showed no specific pathologic change.

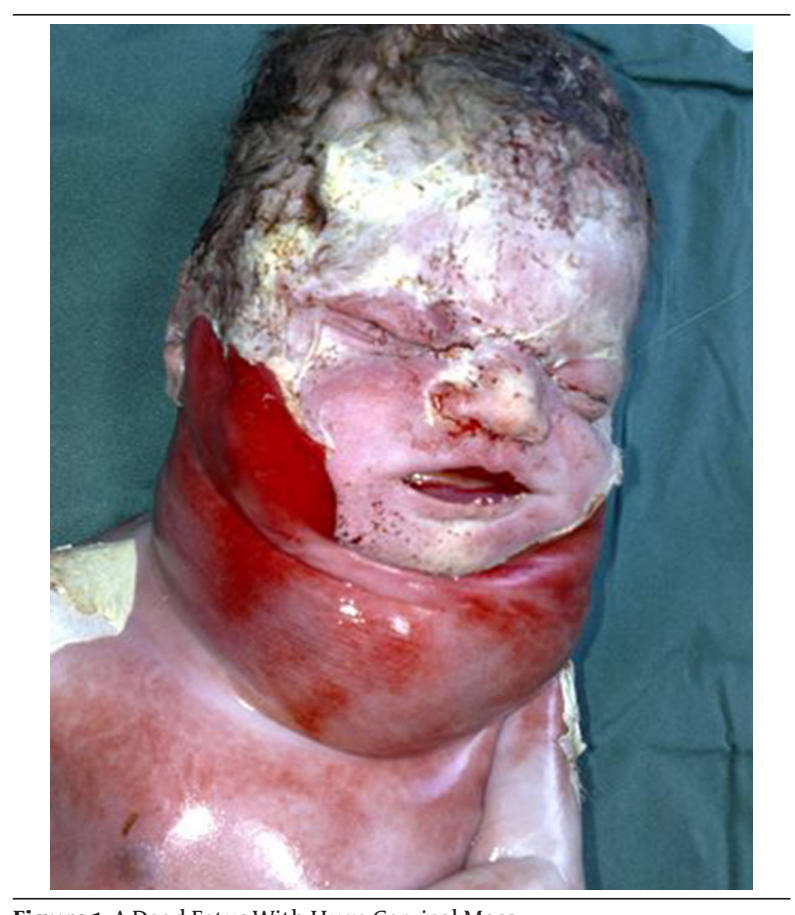

Figure 1. A Dead Fetus With Huge Cervical Mass 

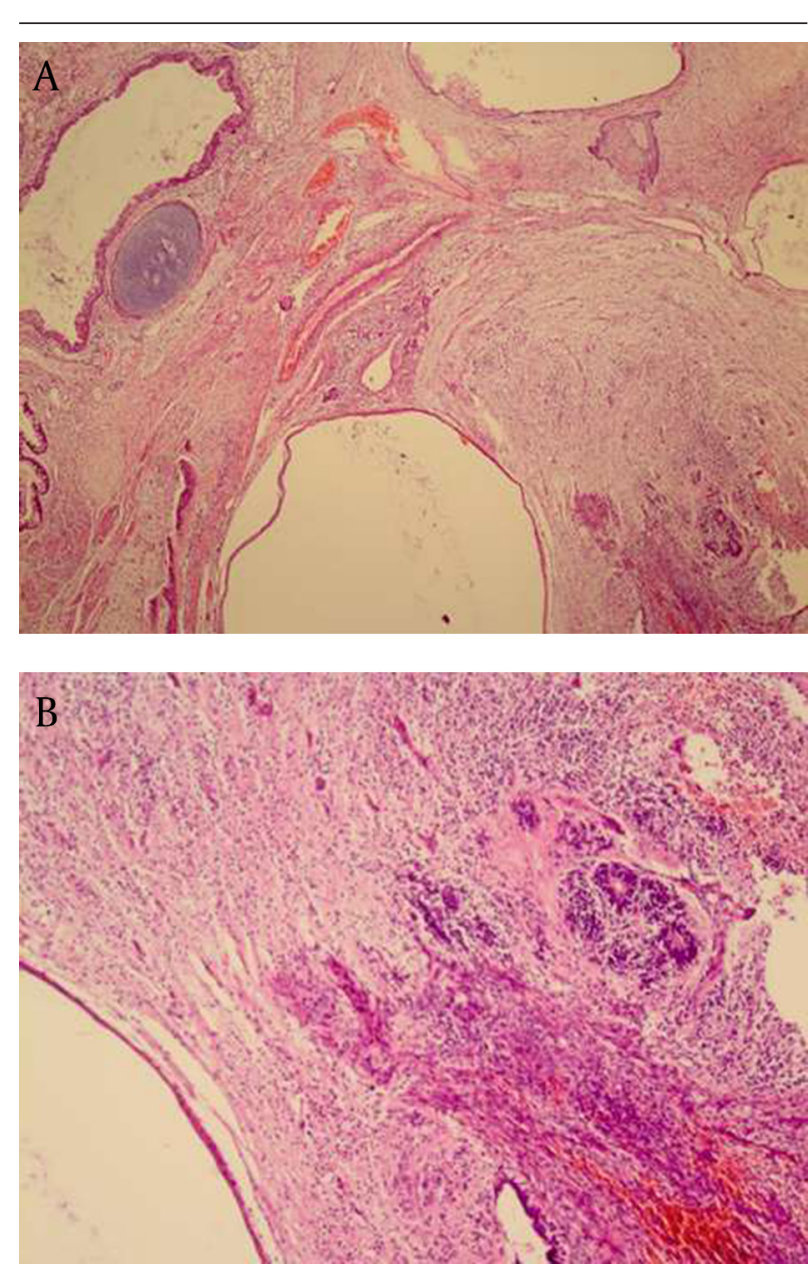

Figure 2. A) Mature component of tumor composed of cartilage, squamous and glandular epithelium, and adipose tissue and smooth muscle fibers. B) Immature component of tumor composed of sheets of small cells with hyperchromatic nuclei, and rosettes formation

\section{Discussion}

Teratomas originate from the pluripotent ectodermal, mesodermal and endodermal germ cells and ectopic embryogenic non-germ cells. Any part of the body may be involved with these tumors, with sacrococcygeal region as the most commonly site (3). Cervical teratoma is a rare neoplasm and its frequency is second to third teratoma arising from the sacrococcygeal region $(4,5)$. Immature teratoma is primarily composed of embryonic appearing neurological or neuroepithelial components more often seen in the cervical teratomas than those at other sites (6). Mostly immature teratoma of the fetus and infants has a favorable prognosis (4). Other congenital anomalies are associated with cervical teratoma reported up to $18 \%$ and involving the urogenital tract, gastrointestinal tract, central nervous system, heart and musculoskeletal system (1). In our case, no congenital anomaly was identified. The differential diagnoses of fetal neck masses include cystic hygroma, congenital goiter or other thyroid masses, thyroglossal duct, branchial cleft cyst and meningomyelocele (3-7). Prenatal sonography is a choice for prenatal screening (8). Detection of dense amorphous calcification in a large predominantly solid neck mass that contain cystic areas in sonography suggested cervical teratoma as a primary choice (7). In our case, thyromegaly or thymoma was suggested in sonography. Maternal polyhydramnios is reported in about one third of prenatally diagnosed cervical teratomas due to the pressure effect of tumor on the esophagus and inability of the fetus to swallow amniotic fluid (3). Moreover, in cases of giant cervical teratomas, polyhydramnios and non-immune hydrops fetalis with certain intrauterine fetal death may develop resulting from obstruction of circulation leading to high-output cardiac failure (6). Therefore, ultrasound examination should be performed in pregnant women developing polyhydramnios for detection of fetal anomaly especially in the cervical region (3). In this case, maternal polyhydramnios is reported without hydrops fetalis. The thyroid gland may be involved in a small number of teratomas in fetus or neonate. These teratomas occupy the soft tissues of the anterior and lateral regions of the neck, resulting in compression of the upper airways. They may infiltrate the thyroid gland or attach to the gland (6). In one of the three cases with cervical teratoma reported by Stefaan J. et al., thyroid gland was not identified (3). In our case, the thyroid gland was not identified in gross and serial sections of microscopic examination. In conclusion, congenital cervical teratomas should be considered in prenatal and postnatal differential diagnosis of neck masses; although, this is a rare condition and may involve the thyroid gland or arise from it.

\section{References}

1. Muscatello L, Giudice M, Feltri M. Malignant cervical teratoma: report of a case in a newborn. Eur Arch Otorhinolaryngol. 2005;262(11):899-904.

2. Laje P, Johnson MP, Howell LJ, Bebbington MW, Hedrick HL, Flake AW, et al. Ex utero intrapartum treatment in the management of giant cervical teratomas. J Pediatr Surg. 2012;47(6):1208-16.

3. Berge SJ, von Lindern JJ, Appel T, Braumann B, Niederhagen B. Diagnosis and management of cervical teratomas. Br J Oral Maxillofac Surg. 2004;42(1):41-5.

4. Potter's P. In: infant and child. 3 ed. Enid Gilbert- Barness, editor Elsevier; 2007. pp.1691-700.

5. Isaacs $\mathrm{H}$, Jr. Perinatal (fetal and neonatal) germ cell tumors. J Pediatr Surg. 2004;39(7):1003-13.

6. Tonni G, De Felice C, Centini G, Ginanneschi C. Cervical and oral teratoma in the fetus: a systematic review of etiology, pathology, diagnosis, treatment and prognosis. Arch Gynecol Obstet. 2010;282(4):355-61.

7. Kangarloo H, Diament MJ, Steckel RJ, Kagan AR. Cervical mass in a fetus associated with maternal hydramnios. AJR Am J Roentgenol.1983;140(3):507-9.

8. Figueiredo G, Pinto PS, Graham EM, Huisman TA. Congenital giant cervical teratoma: pre- and postnatal imaging. Fetal Diagn Ther. 2010;27(4):231-2. 